\title{
XLI. The Greenhouse theory and planetary temperatures
}

\section{Frank W. Very}

To cite this article: Frank W. Very (1908) XLI. The Greenhouse theory and planetary temperatures, Philosophical Magazine Series 6, 16:93, 462-480, DOI: 10.1080/14786440908636529

To link to this article: http://dx.doi.org/10.1080/14786440908636529

$$
\text { 曲 Published online: } 21 \text { Apr } 2009 .
$$

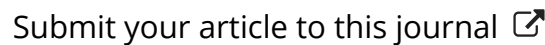

Џ Article views: 9 
good a conducting material as carbon in the spark-gap could be entirely without effect upon the discharge. Rather, it seems likely that the particles present before the first spark were as effective as carbon particles. The writer* has shown clearly that the passage of a spark through an oil does very materially affect the potential required to produce the discharge. So that, valuable as the results of Earhart and Shaw are as representing the actual working conditions which usually obtain, they may not be taken to represent the potentials necessary to produce spark discharges through the distances given, in the pure liquid.

The Brace Laboratory, Lincoln, Nebr., U.S.A.

XLI. The Greenhouse Theory and Planetary Temperatures. By Frank W. Very†.

W HEN Tyndall, as the result of his measures of the absorption of terrestrial radiation by water-vapour, assured us that the removal of moisture from its atmospheric covering would plunge the British Isles into a more than Arctic winter, he directed attention to that which, next to the solar radiation itself, is the most potent factor in terrestrial climate. In a general way this is now universally recognized; but we have not yet arrived at unanimity as to the details of the process by which this protective agency works, or of their applicability to other worlds than ours.

Never having had any experience with gases devoid of convection, nor with solids lacking thermal conductivity, it is perhaps hardly safe to speculate as to bow such substances would or would not behave; but if a guess may be hazarded on the subject of Professor Poynting's ideal greenhouse $\ddagger$, one would suppose that if the background were absolutely nonconducting, unless it were a perfect reflector, in which case it would not be heated at all, the heating effect of solar rays would be increased in something like the proportion of six to one even without any glass. For if we suppose cubical particles, each containing a cubic centimetre of perfectly absorbing and conducting substance, to be maintained in the sun's rays at an excess of $10^{\circ}$ above an initial temperature of $300^{\circ} \mathrm{A}$., and then reduce the thickness of the normal absorbing

* Almy, Ann. der Phys. [4] i. p. 508 (1900).

+ Communicated by the Author.

$\mp$ J. H. Poynting, "On Prof. Lowell's Method for Evaluating the Surface Temperatures of the Planets," Phil. Mag. [6] vol. xiv. p. 749, December 1907. 
layer from $1 \mathrm{~cm}$. to ${ }_{100} \mathrm{~cm}$., the radiating surface being only one-third as much as before, we should have, if there were no loss of heat by convection, three times the thermal effect, giving an excess of $27^{\circ} .5$ by the fourth-power law. But if the thin strip were backed by nonconducting material, the radiating surface would be only one-sixth of that of the cube, while the thermal effect of the same solar radiation would be six times as great, and the excess would be $49^{\circ} \cdot 4$.

Now, where a layer of conducting material of indefinite thickness is to be heated, a day is too short a time to reach thermal equilibrium; but for an infinitesimal layer a day is relatively an infinite time; and since the glass of the greenhouse roof is supposed to let in rays which are transformed at the surface into others which cannot get out, or can get out only in small part, a fraction of the heat from inward radiation must be added to the heat of the infinitesimal layer at each radiant transfer from solid to glass and back, until the temperature of the superficial layer of the solid background is raised so high that it either begins to give out radiations which will penetrate glass, or until the glass emits an equal radiation. If the layer is really nonconducting, or what is the same thing thermally, if its thickness is infinitesimal and its radiation one-sided, or again, if the background conducts but has been exposed to insolation so long that the interior temperature gradient is zero at the surface, a second is long enough for the entire process; or if the glass transmits a little of the infra-red, and a single layer of glass is insufficient to give the maximum effect, additional ones will complete the realization of the maximum temperature in static equilibrium.

Now, as we are not making a world, but only an ideal, or perhaps I should say a transcendental greenhouse, there is nothing to hinder us from piling story on story until we get a greenhouse, let us say, fifty stories high. At first each added story will capture a little extra heat, and this will also go on accumulating until the absorption of the additional sheets of glass for the solar rays is greater than their power to obstruct the passage of radiation of long wave-length, and thus to increase the amount of heat trapped. By making our glass thin enough, however, we can entirely obviate this difficulty ; for by making a greenhouse of one thousand stories, each covered with glass on $\theta$ one-thousandth as thick as that of the ordinary greenhouse, the absorption will be no greater, but the heattrapping will increase up to a certain point in proportion to the number of chambers traversed. Such a structure will have a cumulative action resembling that of a bundle of imperfectly reflecting mirrors, which, if numerous enough, 
reflect as well as a more perfect one. In the present case, the mirror must be supposed to have the peculiar quality of being transparent to the ingoing radiation, and a perfect reflector of the outgoing rays; but with such a mirror, if it were possible to obtain it, there would be no possibility of stopping the accumulation of heat short of a temperature at which penetrating rays of solar quality are emitted.

With absorbent, instead of reflective layers, the temperature can only increase until, in the steady state, the roof radiates out as much as the sun sends in. But since the roof then radiates greatly inward to the background, the latter is receiving the direct solar rays as before there was a roof, and also an equal amount of returned radiation. Hence the thermal effect is doubled. The maximum heat-trapping effect is reached when the inmost layer of the roof and the background reach the same temperature, or when equal amounts of energy are interchanged between these surfaces accurding to Prévost's law of exchanges. The condition is represented in Poynting's equation for the greenhouse effect,

$$
\frac{\mathrm{R}}{\mathrm{S}}=\frac{t+a / n}{t_{1}+(n-1) a_{1} / n}{ }^{*}, \quad . \quad . \quad . \quad . \quad .
$$

if $t=1, a=0, t_{1}=0, a_{1}=1$, and $n=2$, when $\mathrm{R} / \mathrm{S}=2$. This is of course the maximum increment of thermal energy which it is possible to get by means of a greenhouse provided simply with an absorbent covering. The more perfect our heattrapping device becomes, the nearer will be the approach to this ratio. If the number of chambers is not indefinitely great, $n$ must be a little larger than 2 , because each successive chamber in the outward direction is at a little lower temperature, and radiation outward, being towards a colder surface, exceeds radiation inwards.

Having demonstrated the properties of our ideal glass house, I might leave the subject with the remark that at first blush neither it nor Professor Poynting's greenhouse bears the remotest resemblance to our actual complex world. But if the temperature of an ideal planet all depends on hypotheses, let us introduce into our discussion assumptions which make for warmth rather than cold; because evidence drawn from astronomical observations indicates that there is abundant warmth at the surfaces of the more distant planets. I shall endeavour to show that the higher temperatures agree better with facts of observation and with theory.

* $t$ and $a$ denote the transmission and absorption of solar rays (S), and $t_{1}, a_{1}$ the same for ground radiation $(R)$, while $1 / n$ is the fraction of glass radiation sent downward. 
If we look a little further into the matter, perhaps we shall find that my greenhouse raised to the thousandth power is not so much of a reductio ad absurdum as one might suppose; for have we not increasing evidence that matter is really discontinuous, or that it is a form consisting of innumerable chambers? Thermal ensrgy is transferred from molecule to molecule in the interior of a body by a process which involves a time-factor and a discontinuous mechanism. Restore these by giving our background conductivity, and we have again the analogue of the thousand-story greenhouse in the capacity of matter for storing up heat, quite apart from the atmospheric effect.

Short of a duplicate thermal effect, there is no reason why various mechanisms of this sort may not go on increasing in temperature until the emission of short-waved æether vibrations which will penetrate the outermost layers of the protecting roof limits the further acquisition of stored-up energy-that is, there would be none so long as we retain the further property which Professor Poynting assumes, that the atmosphere has no convection. This, however, is too wide a departure from actuality to be permissible in considerations which are to have any bearing on natural phenomena on this earth. Experiment has given the following relative losses of temperature by radiation and by convection: For a small thermometer, heated to $15^{\circ} \mathrm{C}$. in sunshine, about three-tenths of the heat is lost by convection; but in a thin strip of blackened platinum similarly heated, the loss by convection is twice as great as that by radiation. Up to a temperatureexcess of about $15^{\circ} \mathrm{C}$., convective emission from a body of the size of a thermometer-bulb increases rapidly. After this, convection is more nearly a constant proportion of the total loss of heat. At high temperatures, radiation, which varies nearly according to the fourth power of the absolute temperature in solids, is increasingly potent to diminish heat, while the air becomes viscous, and convection is impeded; but for small differences of temperature at moderate temperatures, such as would exist in successive chambers of the multiple greenhouse, convection is of importance in assisting to limit the possible accumulation of heat.

I have tried the effect of successive enclosures of ordinary glass around a sun-thermometer, and find that there is very little heat gained after two or three layers have been added. But probably the progression would be more noticeable if glass of a very thin lamination could be procured. I have indeed used mica with some success.

In an experiment where a black-bulb thermometer in air Phil. Mag. S. 6. Vol. 16. No. 93. Sept. 1908. 2 I 
was exposed to the sun's rays in an enclosure protected by three layers of thin glass, separated by air-spaces, I obtained a reading of $104^{\circ} \mathrm{C}$,, the shade temperature in the air being $35^{\circ} \mathrm{C}$. The altitude of the sun was $50^{\circ}$, and the solar radiation was 1.30 small calories per square centimetre per minute (obtained with a mercury pyrheliometer). The temperature of the superficial layer of the soil was not taken, but is usually about $10^{\circ}$ higher than the air temperature for the given altitude of the sun. Admitting that a layer of soil about $1 \mathrm{~cm}$. deep was maintained at a temperature of $45^{\circ} \mathrm{C}$. by the sun's rays, the retention of heat was augmented by three layers of glass in the ratio

$$
\left(\frac{104+273}{45+273}\right)^{4}=1 \cdot 975 .
$$

This already approaches the theoretical duplicate ratio, and yet nothing has been allowed for heat-trapping by the atmosphere which, with a relative humidity of 42 per cent., must have been considerable. Hence the ground temperature was probably higher than $45^{\circ} \mathrm{C}$.

If we take the excess of surface soil-temperature over airtemperature twice as large, making the surface temperature $35+20=55^{\circ} \mathrm{C}$, , the augmentation by the three layers of glass was

$$
\left(\frac{104+273}{55+273}\right)^{4}=1 \cdot 744 .
$$

Even this gives a smaller heat-retaining power to the atmosphere than it usually possesses ; but except on mountains the excess of surface temperature is seldom greater than $20^{\circ} \mathrm{C}$. Be this as it may, the observation certainly shows that a solar radiation of $1.3 \mathrm{cal} . / \mathrm{cm} .{ }^{2}$ min. is competent to produce a temperature above that of boiling water; and if the solar constant is as large as $3.1 \mathrm{cal} . / \mathrm{cm}^{2} \mathrm{~min}$., which I believe to be probable, the sun can give at the earth's distance a temperature of $\chi^{\circ}$, where

$$
\begin{array}{ll} 
& \chi^{4}:(104+273)^{4}=3 \cdot 1: 1 \cdot 3, \\
\text { or } \quad & \chi=468^{\circ} \mathrm{A} .
\end{array}
$$

Langley * obtained at his mountain camp on Mt. Whitney, in a double hot-box of his design, an excess of $98^{\circ} .5 \mathrm{C}$. above shade temperature. No simultaneous actinometer readings were made; but by interpolation from those of other days

* S. P. Langley, "Researches on Solar Heat," Professional Papers of the Signal Service, No. 15, Chap. 18, p. 166. 
(Table 85, p. 98, loc. cit.), it may be inferred that the solar radiation at the same hour, $1^{\mathrm{h}} 40^{\mathrm{m}}$ P.M., was usually about $1.84 \mathrm{cal} . / \mathrm{cm} .{ }^{2} \mathrm{~min}$. For comparison with my observation, I will suppose the shade temperature to have been the same as mine, giving a reduced hot-box temperature

$$
=35+98 \cdot 5+273=406^{\circ} \cdot 5 \mathrm{~A} \text {, }
$$

whence

$$
\chi=\left\{\frac{3 \cdot 1}{1 \cdot 84} \times(406 \cdot 5)^{4}\right\}^{\frac{1}{4}}=463^{\circ} \mathrm{A} .
$$

These temperatures are also in approximate agreement with that which I have found for the moon at midday $\left(454^{\circ} \mathrm{A} .\right)^{*}$ : The moon, unlike the earth, has a sufficiently prolonged insolation to produce a steady state of temperature, and it has so little atmosphere that very little heat is conveyed to cold parts of the planet through convection currents.

Sun temperatures at planetary surfaces are very greatly modified by atmospheric agencies. In nature the effect of convection is very marked, for the atmosphere acquires its heat mainly by convection of bot air, as well as by evaporation of moisture from a heated surface, and by the distribution of these thermal increments by means of atmospheric circulation.

Atmospheric heating by the absorption of radiation is accomplished with especial efficacy in two layers-one high up, the other near the surface. The multiple greenhouse has therefore a further analogue ; for the incoming solar rays are most efficacious in warming the air in the upper layers of the atmosphere where these rays first meet the atmospheric moisture. A very considerable fraction of infra-red solar radiation is taken out by line and band absorption in the upper air. In passing through the first ten kilometres of air containing appreciable moisture, or what may be called the aqueous atmosphere, certain rays are struck out from the spectrum completely. These rays do not enter into our formula for atmospheric absorption. They have been lost completely and do not reach the earth's surface. Consequently the readings of actinometers which take no account of these lost rays, even after correction for the apparent absorption of the atmosphere determined by high and low sun comparisons, give only the solar radiation, minus an unknown line and band absorption. The solar radiant energy kept back

* F. W. Very, "The Probable Range of Temperature on the Moon," Astrophysical Journ. vol, viii. p. 284, December 1888. 
by those atmospheric constituents which have the first opportunity to exercise absorbent action, is used in increasing the temperature of the upper air, and is probably responsible for a large part of the excess of temperature above that which would be indicated by the adiabatic rate, which has been made known by the records of sounding balloons. The observations of Teisserenc de Bort, who was the first to confirm the existence of this hypothetical warm upper layer of air, have now been extended by the German sounding balloons to a height of 25 lilometres, to which height the aqueous atmosphere certainly sometimes ascends. Undoubtedly the height of the aqueous atmosphere is a variable quantity, subject to wide fuctuations which are partly responsible for the variations of surface temperature. In summer time, the lower part of the aqueous atmosphere stores up a great amount of heat, giving hot waves when descending currents prevail during periods of high baromeier. In winter. on the contrary, the periods of high barometer may be attended by cold waves, because the heavily moisture-laden layer in immediate contact with the surface is swept away, and surface radiation escapes freely; but the cold would then be far more intense if it were not for the presence of the rarer layers of the aqueous atmosphere, which still remain as a warm layer at no great altitude, but are unable to affect surface temperatures much by direct radiation, since the emission of radiation by a gas through a deep layer of its own substance is small on account of its great absorptive power for its own radiation.

The second region of atmospheric heating by absorption of radiation is one near the surface where the infra-red radiations from either land or water are so thoroughly absorbed at certain wave-lengths that these cannot escape except by a slow process of absorption and reradiation many times repeated. In this respect also, the illustration of the multiple greenhouse is again valid, and surface temperatures would accumulate until they became unbearable, did not convection overturn the atmospheric heat-retaining structure.

The retaining action of an atmosphere for surface heat cannot be represented by so simple a device as that of a thin sheet of perfectly conducting glass, having the same temperature at its upper and lower surfaces. It seems to me that in part Professor Poynting's argument is irrelevant as regards the problem of the surface temperature of a planet without the inclusion of further details. If Professor Poynting will turn his great mathematical ability to the consideration of some of the numerous alternative hypotheses, it seems pro- 
bable that a much wider range of possibilities may appear. Even if the complete differential equations cannot now be attempted, the problem can be taken up in sections, as he has done for a part of the process, investigating one influence after another separately, but of course recognizing that these will have mutual modifications which must be considered in the final summing up.

Take, for example, the climatic influences involved in the evaporation and condensation of water. M. Liais many years ago estimated that this process has a very large part in determining terrestrial thermal conditions. I will suppose, in illustration, that we seek the effect of this factor on the climate of Mars. Assuming that the barometric pressure is much smaller on Mars, a supposition for which there is good reason, evaporation of moisture at a given temperature will be more rapid, and condensation more difficult than here. Consequently, at that temperature, more moisture can exist in the atmosphere of Mars, diffusing to a great beight, and still the relative humidity will remain low*. The smaller relative humidity diminishes the strength of the broad diffuse bands of aqueous absorption, but does not affect the line absorption. Since condensation of moisture is favoured by the presence of free ions to serve as nuclei of condensation, we should like to know something as to this condition on Mars. We do know that here, with abundance of moisture in the air, rain does not fall unless some widespread general condition of the atmosphere, which appears to be associated with its electrical state, is added to the other favouring factors. Telescopic observation of Mars shows a very considerable degree of persistence in the surface markings which indicates comparative absence of obscuring cloud, that is to say, there is less liability of rain, moisture remains long in the air, and the continuity of the protecting aqueous envelope is more complete. Though less heat is received, the final result, if more is stored, may be not very different in the two planets so far as this factor is concerned.

Professor Lowell $\uparrow$ emphasizes the importance of albedo in

* Since this was written, Mr. Slipher has obtained at the Flagstaff Observatory spectrograms of Mars showing intensification, not of the comparatively feeble " rain band" near D which has been the subject of much contention in the past, but of the much more powerful water-vapour band " $a$ " in the extreme red. I have had the privilege of examining the original photographs, and can testify to the certainty of the evidence they bring of the presence of water-vapour in the atmosphere of Mars.

t Percival Lowell, "Temperature of Mars," Proc. American Acad. Arts and Sciences, vol. xlii. p. 65l, March 1907. 
determining a planet's temperature, and shows that a planet's albedo is largely atmospheric. This being so, since about a third of the sun's radiant energy resides in the visible spectrum where selective scattering is most effective, the earth, with its greater albedo, receives a smaller proportion of heat at its surface than Mars on this account. In the visible region of the spectrum, the radiant absorption by gases is small, and the blanketing action of the atmosphere nil, because there are no rays of this wave-length emitted by its heated surface; but the larger proportion of heat received from this part of the spectrum by the ground of Mars produces, if the absorbent and conducting properties of the surface are the same, a relatively larger radiation of infra-red rays outward, which is at the same time proportionally better preserved.

Land temperatures on this earth exceed those over water. Mars has the land climate. The heat-storing powers of the soil are also greater with a dry climate. Again, the conditions on Mars favour a greater thermal effect from the same amount of solar radiation. That the cumulative effect of so many factors acting in one direction can largely overpower a deficiency of that due to remoteness from the sun, is a conclusion which is at least permissible; and if computations founded on reasonable assumptions lead to results which are in good agreement with the seasonal indications deduced from the fluctuations of a snow-cap whose existence is far more probable than some of the hypotheses made in arguments against its possibility, it seems to me that the balance of the evidence inclines strongly towards Professor Lowell's conclusions.

In treating the subject of atmospheric selective depletion of radiation, a more accurate result might be obtained by treating the diffraction by air molecules, and the selective reflexion from dust separately; but without going into this refinement, an approximate knowledge of the reflective depletion of the solar rays falling on an air-covered planet can be reached if we admit that the transmission, so far as it depends on reflexion, varies as $t_{r}^{\varepsilon}$. where $t_{r}$ is the reflective transmission for rays at the zenith, and $\epsilon$ is the air mass. The depletion will then be $1-t_{r}^{6}$, and the total reflective depletion for the entire surface of the sunlit atmosphere is

$$
\Sigma\left(1-t_{r}^{\epsilon}\right) \times \Delta \cos \zeta
$$

where $\zeta$ is the sun's zenith distance. For $t_{r}=0^{*} 6$,

$$
\Sigma\left(1-t_{r}^{\kappa}\right) \Delta \cos \zeta=0.677 \text {, }
$$


which corresponds very nearly with the depletion for a solar altitude of $27^{\circ}$.

Bearing in mind that the discussion at this point does not relate to the line and band absorption which follow different laws, the value of $1-t_{r}$, or the atmospheric depletion by diffraction and selective or scaltering reflexiou, may be approximately as follows :-

By taking the spectral energy-curves given in my reduction of the Mount Whitney observations *, and making a further reduction to sea-level, Professor Lowell obtains $\dagger$ for the light reflected by the air from sea-level upwards, the fraction 0.74 . In the reduction to sea-level, observations made at different places and times, and with different instruments, were used, which is objectionable, but unavoidable, It is also desirable to differentiate the depletion in the infra-red into several kinds. I do not know that 0.74 is too large for the air-reflexion of visible rays, but we shall be taking a lower limit if we make it 0.6 . It cannot be as much for the air above clouds. As clouds hang at very different altitudes in the air, noexact statement can be made as to the light reflected by the air before the sun's rays reach the cloud level. Let it be 0.3 , and the reflexion from clouds 0.72 , as has been given by observation. Then the light reflected from a cloudy area of the earth's surface will be $0.3+(0.72 \times 0.7)=0.804$, giving the albedo of a balf-clouded earth, $\frac{1}{2}(0 \cdot 6+0 \cdot 8)=0 \cdot 7$. Only a small amount of infra-red radiation is reflected by the air. Let us say 0.2 for clear air, and 0.1 for the air above clouds. But the clouds also do not reflect the infra-red rays as well as they do the visible rays; for I have been able to determine the position of the sun bolometrically through clouds so thick as completely to hide itfrom the eye. Allowing that clouds may reflect the infra-red rays half as well as the luminous ones, infra-red reflexion from a cloudy area is $0 \cdot 1+(0.36 \times 0 \cdot 9)=0 \cdot 424$. One third of the rays being visible, the total reflexion over cloud is

$$
(0.33 \times 0.804)+(0.67 \times 0.424)=0.549 .
$$

The reflexion by clear air is

$$
(0.33 \times 0.6)+(0.67 \times 0.2)=0.332 \text {; }
$$

and the mean reflexion from a half-clouded earth for the entire spectrum is $\frac{1}{2}(0.549+0.332)=0.44$, giving $t_{r}=0.56$.

* U.S. Wenther Bureau Publication No. 254, "The Solar Constant," fig. 1, p. 21 .

$\dagger$ Proc. American Academy of Arts and Sciences, vol. xlij. p. 656 M arch 1907. 
Professor Poynting objects* to Lowell's value for the reflexion of light by the air as being too large. "On another point," he says, "common observation appears, at any rate at first sight, to contradict Professor Lowell. He assumes that the loss in the visible spectrum radiation in its passage through the atmosphere is practically all due to reflesion, and he puts it down as about 0.7 of the whole in clear sky. If this were true the reflexion from the sky opposite to the sun would I think be vastly greater than it is. White cardboard reflects diffusely about 0.7 of sunlight. But when a piece of white cardboard is exposed normally to the sun's rays, it is several times brighter than the cloudless sky."

This is true if the white surface is compared with a deepblue sky far away from the sun; but it is not always true for the sky near the sun, and the upward reflexion towards the sun is probably nearly proportional to the downward reflexion when the sky is only slightly hazy. I tried the following experiment, on a day with hazy blue sky, whitish near the horizon:-The sun being at an altitude of $20^{\circ}$ and shining on fresh snow, I measured with a werlge-photometer the visibility (a) of a wide area of bright background, and (b) that of a narrow dark bar projected on the same background, getting for

Sky near the Sun............. (a) 13.5 mag. (b) 13.0 ,

Sky at horizon, azimuth $45^{\circ}$ from sun .. $120 \quad 115$,

Snow in sunshine.............. $12 \cdot 0 \quad 11.5$.

Taking the ratio of brightness corresponding to the difference of magnitudes, the sky near the sun was four times as bright as the sky at the horizon; but the latter was as bright as the sunlit snow. I confess that I was surprised at the last equality, for the sunlit snow gave me the impression of greater brightness; but this impression is no doubt due to the absence of colour in the snow, while the blue tint of the sky gives one a conception that the sky-light is dimmer than it really is. The sky at the horizon was half a magnitude brighter, or $1 \cdot 6$ times as bright as the sky at an altitude of $20^{\circ}$ and azimuth $90^{\circ}$ from the sun.

A repetition of the experiment on another occasion gave me the following results:-The sky was a pale blue with a few fracto-cumuli, but without appreciable cirrus-haze. The observations were made through a slit $3 \mathrm{~cm}$. wide, viewed at a distance of 1 metre by means of the wedge-photometer. The point in the sky selected for measurement was distant from the sun about four diameters, or far enough away for

* Phil. Mag. [6] vol. xir. p. 750. 
the brightness to be nearly uniform. Mean altitude of $\operatorname{sun}=28^{\circ}$.

$$
\begin{aligned}
& \begin{array}{c|c|c|c}
\text { Sky, 13.0 mag. } & \text { Srow 11.7 mag. } & \text { Sky, } 12.8 \text { mag. } & \text { Snow } 11.8 \text { mag. } \\
13.4 & \text { in } 11.4 & 13.0 & \text { in } 11.6 \\
13.2 & \text { Sun, } 11.5 & 13.1 & \text { Sun, } 11.7 \\
13.0 & 11.3 & 13.1 & 11.8 \\
12.8 & 11.8 & 130 & 11.8 \\
\text { Mean 13.08 } & \underline{11.54} & \underline{13.00} & \underline{11.7 t}
\end{array} \\
& \text { Difference of maqnitude, 1st set, Sky }=+1 \cdot 5+\mathrm{mag} \text {. } \\
& \text { " } " \text { 2nd " " +1.26, }
\end{aligned}
$$

The mean difference is sky $=+1.4$ magnitude, or the sky had 3.63 times the brightness of sunlit snow.

With a clear blue sky, I found for-

$\begin{aligned} \text { Sun's altitude } & =30^{\circ} \text {, snow } 1.6 \text { times brightness of sky, } \\ , " \quad=10^{\circ} \text {, sky } 1.35, ", & \text { snow. }\end{aligned}$

It appears from these comparisons that the discrepancy between the brightness of the sky and of a white sun-illumined surface is not as great as one is apt to guess.

If one stands with his back to the sun and compares the light reflected from the sky with that from a piece of white paper, the former comes principally from remote depths of air, and has suffered further depletion in returning thence to the eye. It is therefore not to be expected that the air should reflect as well as a piece of white paper held close to the eye.

It will no doubt be agreed that the depletion suffered by the sun's rays in the middle of the visible spectrum is not due to telluric absorption-bands, but results solely from diffraction and scattering reflexion. I measured the light from the sun, when it was about to set, and compared its intensity in the green of the spectrum with that which the same region had when the sun was $30^{\circ}$ high. The intensity of green light from the nearly setting sun was 0.0000065 times that at the higher altitude. The relative air-masses were $2 \cdot 0$ and $24 \cdot 4$, that at the zenith being unity. The zenithal transmission corresponding to that for air-mass $24 \cdot 4$ was therefore

$$
(0.0000065) \frac{1}{2+4-2 \cdot 0}=0.587 \text {, }
$$

and the transmission at a middle altitude of $30^{\circ}$ was $(0.587)^{2}=0.345$, or the depletion equal to 0.655 , which must 
be further increased in a summation for the entire exposed hemisphere, becoming $0 \cdot 690$. This value is too small, because the reflexion from dust is to a certain extent -independent of the air mass; but it represents fairly well the reflective depletion for the entire spectrum.

Although the above is only a single observation which would have to be repeated on many different occasions to give a thoroughly reliable result, it is at least something better than a guess. To this depletion must be added the band absorption which chiefly affects the infra-red spectrum, and may be taken for moderate altitudes above the horizon in temperate latitudes as $0.3 \times 2 / 3=0.2$, but which is probably twice as great for the entire earth with its moist tropics.

The radiation received from a zenith sun is $(\mathrm{A}-\mathrm{B}) \times t_{r}$, where $A$ is the solar constant, and $B$ is the band absorption. If $A$ is 3 calories per square centimetre per minute,

$$
(\mathrm{A}-\mathrm{B}) t_{r}=(3.0-0.6) \times 0.56=1.34 \text { cal., }
$$

a quantity which is not often exceeded at sea-level.

In regard to selective, reflective depletion of the solar rays, it is clear that radiation of every wave-length undergoes more or less superficial reflexion at the outside surface of suspended particles of dust, ice crystals, \&c. This differs in no wise from the reflexion at the surface of a mirror, although if the particles be many-faceted, the rays will be reflected in many directions and may not be distinguishable from the diffuse reflexion from internal bubbles or other discontinuities in a transparent but turbid medium. This surface reflexion is a general one, or in the visible spectrum we should call it a colourless one. If the rays penetrate a little way into the substance before being reflected, certain rays may be absorbed, giving selective reflexion, which in the visible spectrum we call coloured reflexion. Melloni coined a special word for the selection of invisible rays, labelling it " thermochrose," but the distinction is not needed. Finally, molecules and particles whose dimensions are small relatively to the wave-length give selective diffraction which varies progressively with the wave-length. I have used the word depletion to cover losses by all of these processes without individual specification. Any part of the solar radiation which is reflected back into space can have no effect on planetary temperature. This precept is so obvious that it seems strange that it should be overlooked.

In considering the effect on climate it is not so easy to decide how we ought to treat the band absorption. So far as absorption occurs in the upper air, it raises its temperature, and this the more on account of the low density of the air at 
great altitudes. Any extra warmth in the higher layers of the atmosphere must have some effect in raising the temperature of lower ones. Heat transferred from earth to air is partly lost as radiation from air and cloud before being returned to earth. Whether the loss is counterbalanced by heat from absorption of solar rays in the upper air brought down to earth in descending air-currents as an accession to the potential temperature which is represented by the work stored up in a previous expansion, it might be difficult to determine; but quite a large part of the band absorption may be indirectly useful in enhancing surface temperatures. Professor Lowell cuts the Gordian knot by an ingenious method which dispenses with a knowledge of these data. Before passing to it, I wish to consider what Professor Pornting calls the "greenbouse effect" in planetary temperatures a little more in detail.

In Poynting's equation for the atmospheric greenhouse effect, $1 / n$ is the fraction of the radiant energy absorbed by the air which is returned downwards. Of $n$ Professor Poynting says: "I do not see how to estimate the distribution of the radiation from the air between the upward stream into space and the downward stream to the surface." This being so, I propose to substitute values for the other factors which are less problematical and determine $n$. Following Lowell, Professor Poynting has assumed $a_{1}=0.5, a=0.325, t_{1}=0.5$, $t=0 \cdot 42$. From (1) we obtain

$$
n=-\frac{a_{1} \mathrm{R}+a \mathrm{~S}}{t \mathrm{~S}-t_{1} \overline{\mathrm{R}}-a_{1} \mathrm{R}} \cdot \text {. . . . . . }
$$

Let $\mathrm{S}=3 \mathrm{cal} . / \mathrm{cm} .{ }^{2} \mathrm{~min} .=2 \cdot 1 \times 10^{6} \mathrm{ergs} / \mathrm{sec}$., which distributed over the whole earth becomes one fourth of this, or $5.25 \times 10^{5} \mathrm{ergs} / \mathrm{sec}$. Taking the mean temperature of the whole earth as $\theta=288^{\circ} \mathrm{A}$.,

$$
\mathrm{R}=\sigma \theta^{4}=5.32 \times 10^{-5} \times 288^{4}=3.66 \times 10^{5} \mathrm{ergs} / \mathrm{sec} .
$$

Substituting these values in (2), $n=2 \cdot 43$, that is, less than half of the atmospheric radiation comes downwards; but the determination probably has little or no significance, because the storing effect of the atmosphere is not a purely radiant effect. In fact the radiation from the atmosphere to the earth is comparatively small, as I have shown in my "Atmospheric Radiation." Although much of the energy of terrestrial radiation absorbed by the air comes back to the ground, it does so largely by modes other than radiant, such as the condensation of water-vapour into rain. The green- 
house effect, using the term in a very wide sense, may still be attained, but by a very complicated process.

If we apply Poynting's equation in its strictly limited sense to my hot-box experiment, its use becomes legitimate. The following approximate values are close enough for illustration: Take $t=0 \cdot 8, a=0 \cdot 2, t_{1}=0, a_{1}=1,\left(\theta_{8} \mid \theta\right)^{4}=1 \cdot 744$. Substituting in the equation

$$
\begin{aligned}
& \left(\theta_{8} / \theta\right)^{4}=\frac{t+\alpha / n}{t_{2}+(n-1) a_{1} / n}, \quad . \quad . \quad . \\
& n=1.944 / 0.944=2 \cdot 06
\end{aligned}
$$

which is in substantial agreement with Professor Poynting's supposition that one half of the radiation of the glass goes downward, although, of course, my experiment does not quite fulfil his requirement of a greenhouse of indefinite extent.

In the application of the formula of Arrhenius to the cloudcovered half of the earth's surface, Poynting says that Lowell " finds that this half only receives 0.2 of the radiation which the clear-sky half receives. The surface temperature uhder cloud should therefore be only $\sqrt[4]{0 \cdot 2}=0 \cdot 67$ of that under clear sky. If the latter is $300^{\circ} \mathrm{A}$, the former is only about $200^{\circ} \mathrm{A}$. Common observation contradicts this flatly, for the difference is at most but a few degrees." This only illustrates the inadequacy of a consideration of the problem from a purely radiant aspect. Suppose that the clouds are so dense that only one per cent. of solar radiation reaches the surface. Must we presume that the temperature will immediately fall to near the absolute zero? Not at all. The true surface temperature cannot be found unless the heat received be linked with the heat lost. This Lowell does, obtaining from his analysis of day and night temperatures on the earth a value of the relative emissivity of the earth, and finding that only about 16 per cent. of the heat received from the sun's rays in the day-time is lost by radiation into space in the twenty-four hours. What becomes of the rest? Obviously if so much heat is retained, the earth must be getting hotter. The answer to the question is that the earth does get hotter in summer, but the extra heat is lost in winter; and the entire process requires an estimate of the heat transferred from the summer to the winter hemisphere by the winds of the globe.

On p. 749 (loc. cit.) Professor Poynting says that Lowell "pays hardly any attention to the 'blanketing effect, or, as I prefer to call it, the 'greenhouse effect' of the atmosphere." 
It is true that Professor Lowell does not consider the greenhouse effect analytically and obviously, but it is nevertheless implicitly contained in his deduction of the heat retained, obtained by the method of day and night averages. The method does not specify whether the heat is lost by radiation or by some more circuitous process; and thus it would not be precise to label the retaining power of the atmosphere a "greenhouse effect" without giving a somewhat wider interpretation to this name. If it be permitted to extend the meaning of the term to cover a variety of processes which lead to identical resnlts, the deduction of the loss of surface heat by comparison of day and night temperatures is directly concerned with this wider "greenhouse effect." The method employed by Professor Lowell is a good one, for it dispenses with a detailed knowledge which we may hope to possess eventually, but which, when found, cannot be incompatible with the conclusions deduced by the less circumstantial method. Thus the objection which Professor Poynting makes to the method where be says that Lowell "neglects both the surface radiation reflected down again and the radiation downwards of the energy absorbed by the atmosphere," seems to me to be, on the contrary, one of the strong points in its favour; because for its use it is not necessary that we should know these details. The transmission by cloud for solar and terrestrial radiation may not be the same; but if a complex of radiant and convectional processes which cannot be represented by so simple a formula as that of Arrhenius, gives a virtual equality of depletions under clondy or clear sky, which can be represented by the statement that, though less heat is received under cloud, less is also lost and in about the same proportion, the precise part assigned to each fraction of the process does not affect the argument.

Revorting to my simile of the bundle of mirrors, transmitting some rays and perfectly reflecting others, may it not be possible to realize it? It is known that there are some transparent substances, such as quartz, which have bands of metallic reflexion in the infra-red spectrum. If there were gases in which such bands were sufficiently numerous in the infra-red without too seriously encroaching upon the visible part of the spectrum, it would be possible to obtain almost any increment of tempierature at the surface of a planet covered by such selectively reflecting gases.

The major planets have atmospheres containing unknown and highly absorbent gases which produce bands in the visible spectrum, increasing in strength with the distances of the planets from the sun. These bands invade the red end of 
the spectrum, but diminish in width and blackness towards the shorter waves, and die out near the middle of the visible spectrum. This suggests that there may be present in the atmospheres of these planets substances having very numerous and intense absorption bands in the infra-red; and if these bands are so strong that they give metallic reflexion for surface radiation, even the small amount of energy in the solar radiation on Neptune can be so largely stored up that a high surface temperature is maintained by the accumulated heat. The great inclination of the axes of the two outer planets, whereby large parts of their surfaces remain for long years in a state of arctic night, may serve in this case as a safety-valve by the transferring of heat to cold regions by atmospheric circulation, thus preventing too great an accumulation in the sunward zones. I believe that this, though problematical, is a plausible explanation of the high temperatures which exist on the major planets, as evidenced by the vigour of their atmospheric circulation, the formation of cloud-belts, \&c., which has driven some astronomers to maintain that these planets are much younger than the earth, although there is no reason for such a supposition, and much weighty evidence to the contrary.

If in equation (1), distinguishing between relative losses by reflexion and absorption, so that the completed equation (vide Poynting) is

$$
\mathrm{R}=t \mathrm{~S}+r_{1} \mathrm{R}+\frac{1}{2}\left(a \mathrm{~S}+a_{1} \mathrm{R}\right), . . .
$$

we put $t=0.1, r=0.7, a=0.2, a_{1}=0.01, r_{1}=0.99, t_{1}=0$, we find for $n=2$,

$$
\mathrm{R}=40 \mathrm{~S} \text {. }
$$

I will take for the maximum or steady temperature in sunshine at the earth's distance a mean of my three values, $1 / 3(468+463+454)=462^{\circ} \mathrm{A}$. Then, since the inverse square root of Neptune's distance from the sun is $0 \cdot 18$, the sun temperature at Neptune is $462 \times 0.18=83^{\circ} \mathrm{A} .=\theta_{s}$, and

$$
\theta_{\mathrm{N}}=\sqrt[4]{40 \times\left(\theta_{\mathrm{s}}\right)^{4}}=323^{\circ} \mathrm{A} .=+50^{\circ} \mathrm{C} \text {; }
$$

that is, it is possible for Neptune to have a torrid climate from the solar rays alone without owing anything to its own internal heat, and this without requiring any exceptional action of its atmosphere on luminous rays, or such as would be noticed in telescopic examination.

After what precedes, it will be seen that we cannot compare the heat-retaining powers of the two planets, unless we have reason to believe that the heat-retaining process is similar 
in the two bodies considered. Thus we cannot compare the greenhouse effect for the earth and Neptune; but Mars and the earth appear to resemble each other so nearly that Professor Lowell's method for obtaining the surface temperature of Mars is appropriate.

In this method as applied to the earth, it is assumed that clouds transmit 20 per cent. of terrestrial radiation from a surface at sea-level, and clear air 50 per cent., values which are close enough as approximations. The mean of these being $0 \cdot 3 \overline{5}$, if $y$ is the radiant energy received at the earth's surface, and $e$ the relative emission of radiation from that surface, the heat retained is equal to that radiated from the surface, or

$$
\sigma \theta^{4}=\sigma(288)^{4}=y(1-0.35 e) . . . \quad . \quad .
$$

Lowell introduces into this equation different values obtained by observation of the loss of temperature by nocturnal radiation with clear sky, and also under a cloudy sky; and thus obtains for $e$ the value, $e=0.4634$, whence

$$
\sigma(288)^{4}=0 \cdot 838 y .
$$

By the ratio of retained heat deduced from my hot-box experiment, when compared with a possible maximum retention of heat of 2 to 1 , we get

$$
1 \cdot 744 / 2 \cdot 000=0 \cdot 872 y \text {. }
$$

We may conclude that something like 85 per cent. of the heat received from the sun's rays is retained at the earth's surface through the greenhouse effect of the atmosphere.

For the whole carth with its moist tropies, and including marginal zones where the solar rays pass through a great thickness of absorbent, the band absorption is probably about 0.4 . If 0.7 of the solar radiations are reflected from the hemisphere exposed to the sun's rays, and if of the $(1-0.7) \times(1-0.4)=0.18$ received, 85 per cent. are retained, the temperatures involved become according to the fourthpower law

whence $\theta_{s}=460^{\circ} \mathrm{A}$.

$$
\begin{aligned}
\sigma(288)^{4} & =\sigma\left(\theta_{s}\right)^{4} \times 0.18 \times 0.85 \\
& =\sigma\left(\theta_{s}\right)^{4} \times 0.153,
\end{aligned}
$$

In the last part of his paper, Professor Poynting makes "an attempt to represent the effect of day and night on the temperature of the earth"; but as he neglects the conduction of the ground, and assumes that "the surface has reached an equilibrium between receipt and expenditure of radiation," a condition which is never attained on the earth, since also he considers that a large amount of radiation is absorbed by the air, "nearly 1 with the setting sun," which 
is not the case, and as he then introduces hypothetical values of $n$, the reciprocal of the fraction of air radiation radiated downwards, the attempt cannot be regarded as successful. The band absorption which alone communicates heat to the air directly, remains but a fraction of the total depletion even at sunset. I doubt if the band absorption ever exceeds 0.5 .

Since no reasonable modification of the constants will enable the simple formula for a glass greenhouse to fit the atmospheric conditions on our earth, the formula is inapplicable to problems of planetary temperature without extensive modifications, except perhaps in the conceivable case of a special reflective atmosphere.

Westwood, Massa.husetts, February 7, 1908.

XIII. The Production of Small Variable Frequency Alternating Currents suitable for Telephonic and other Measurements. By B. S. Cohen *.

[Plates XVI.-XIX.]

INTRODUCTION.-The devices described in the latter part of this paper are the outcome of experiments carried out in the Investigation Branch of the Engineering Department of the National Telephone Company, in order to obtain suitable alternating currents both of simple and complex wave form to act as substitutes for the voice in telephonic measurements. Although primarily designed for this purpose, it is considered possible that the apparatus to be described is capable of more extended use.

A short summary of the methods known to the author of obtaining small alternating currents of the kind suitable for telephonic measurements may prove of interest as an introduction.

Statement of the problem.-The ordinary telephonic current is a few milliamperes at a potential of about 2 to 10 volts and is of complex wave form. The frequency of the fundamental harmonic generally lies between 100 and 300 complete periods per second, i.e. it varies from 103 - to $300-$, the highest harmonic having a frequency of $4000 \sim$ to $5000 \sim$, although all harmonies above 1500 - are comparatively unimportant. The average frequency of the whole wave is about $800 \sim$. From consideration of these data it follows that the ideal device for supplying such alternating current is one which will give currents of any frequency lying between 100 to $500 \sim$, singly or in combination. The output of this

* Communicated by the Physical Society : read May 22, 1908. 